\title{
Characterization of Flower and Fruit of Sugar Palm in Pandegelang and Serang Districts, Banten Province
}

\author{
* Nurmayulis Nurmayulis \\ Department of Agroecotechnology \\ Faculty of Agriculture, \\ University of Sultan Agung Tirtayasa, \\ Indonesia Center of Execelenc for Food \\ Security (I-CEFORY), UNTIRTA \\ (Local Food Innovation) Serang City, \\ Indonesia \\ *nurmayulis@untirta.ac.id \\ Susiyanti Susiyanti \\ Department of Agroecotechnology \\ Faculty of Agriculture, \\ University of Sultan Agung Tirtayasa, \\ Indonesia Center of Execelenc for Food \\ Security (I-CEFORY), UNTIRTA \\ (Local Food Innovation) \\ Serang City, Indonesia
}

\author{
Meilani Yusi \\ Magister of Agriculture student, \\ University of Sultan Agung Tirtayasa \\ Serang City, Indonesia
}

\author{
Ismingsih Sulastri \\ Department of Agroecotechnology \\ Faculty of Agriculture, \\ University of Sultan Agung Tirtayasa \\ Serang City, Indonesia
}

\begin{abstract}
Sugar palm (Arenga pinnata Merr,) has a high economic value and have the potential to export and can be processed into various food and non-food products. Sugar palm is a versatile tree because all parts of the tree can be used, such as a trunk, palm sap, leaves, etc. Characterization of local sugar palm tree has been carried out in Pandeglang and Serang districts (Banten Province) which used as breeding material to create make a better sugar palm in the future (superior variety). The study used a survey method based on guidelines for plantation germplasm management from the Indonesia Center for Estate Crops Research and Development. The results showed the diversity in male and female flowers and seeds of a sugar palm tree at both of two districts. The diversity of flower phenotypes on sugar palm tree seen in the number of male and female inflorescences, the height of male and female inflorescences, the length of male and female inflorescences, the circumference of male and female inflorescences, the length series of male and female inflorescences. The diverse appearance of male palm fruit found in the length of the palm fruit, the fruit circumference, and the fruit weight. The conclusion is that the accessions used in this study showed variations in the appearance of male and female flowers and fruit from the sugar palm tree accessions observed.
\end{abstract}

Keywords: Arenga pinnata, Banten, Characterization, flower, fruit, phenotype

\section{INTRODUCTION}

Indonesia is a country that has high biodiversity. This is one of the most potential strategies in order to increase productivity, quality and competitiveness of crop commodities through a plant breeding approach. Greater attention to existing germplasm needs to be improved, especially local varieties through efforts to manage germplasm optimally in the form of inventory (collection), data collection (documentation) and conservation (conservation) activities which are then followed by efforts to identify characters important through systematic characterization and evaluation activities [1].

One of the germplasm that currently needs to be considered and developed in Indonesia is the palm tree (Arenga pinnata Merr.). Given that sugar palm has a production function to produce various commodities that have high economic value and export potential if it is cultivated seriously, because all parts of the plant can be processed into various food and non-food products.

In Banten Province in 2015, the area of palm trees was 2,954 Ha with the largest population in the Lebak District $2,454 \mathrm{Ha}$. Palm sugar as one of the specific local superior commodities of Banten by some people is still believed to be technically unable to be cultivated, the development of the number of palm trees is highly dependent on the "performance" of the ferret weasel [2].

The main problem in the development of sugar palm plants, including in Banten Province, is that the use of palm trees has been going on for a long time, but their development into agribusiness commodities is relatively slow, because some of the existing palm plants grow naturally or have not yet been cultivated. Sugar palm cultivation has not been widely implemented, because so far what has been carried out is still limited to handling harvest, post-harvest and marketing aspects.

According to [3], the most urgent need at the moment is the supply of high-quality seeds from high-yield palm trees. Until now, the source of quality palm sugar seed is not yet available while the genetic erosion of the sugar palm plant runs so fast. So many areas of sugar palm plants that have been converted for other purposes. If this is left continuously without rescue measures then over time the type of quality sugar palm will become extinct. This was also experienced in Banten Province. 
Quality palm seeds will be obtained through a gradual and continuous activity that is preceded by Exploration, Identification and Characterization activities then followed by selection and collection. Exploration is the search for plant genetic material, in the form of genotypes, cultivars, plant clones from nature such as plants that exist in farmers. The purpose of a germplasm exploration is to introduce the genetic diversity of existing germplasm collections. Furthermore, the purpose of germplasm collection is to collect genes contained in plant species that will be very useful in genetically improving the cultivars of a plant [3].

According to [4], exploration is the search for plant genetic material, in the form of genotypes, cultivars, plant clones, from nature such as plants that exist in farmers or from laboratory or individual collections. The purpose of a germplasm exploration is to enrich the genetic diversity of an existing germplasm collection.

Identification is an activity of characterizing all traits possessed by sources of plant genetic diversity. Identification is carried out to find and recognize the taxonomic characteristics of diverse individuals and include them in a taxon [5].

Phenotype is a characteristic (both structural, biochemical, physiological, and behavioral) that can be observed from an organism that is governed by genotype and environment and their interactions. The definition of phenotype includes various levels in the expression of genes of an organism. At the organism level, phenotype is something that can be seen, observed and measured according to the nature or character possessed. The phenotype is partly determined by the genotype of the individual, partly from the environment in which the individual lives, time, and on a number of traits according to the interaction between genotype and environment. Time is usually classified as an environmental aspect [6].

The purpose of this study is to characterize the interest and fruit of the sugar palm germplasm in Pandegelang and Serang District, as an initial initial step in obtaining material for superior palm sugar assembly in the future.

\section{MATERIALS AND METHODS}

The research sites were in 2 regencies in where Pandeglang regency was precisely in Cigeulis district, Cibaliung district, and Cimanggu district, Serang regency was in Mancak district, Gunung Sari, and Ciomas district. This research was conducted from September to December 2018.

Materials and equipment used were: sugar palm plants, meter, digital scales, knives, self adhesive labels, brand boards, stationery, labels, transparent bags, raffia ropes, gloves, identification matrices and characterization of plants and digital cameras. The first stage was exploration with a survey method where random sampling was carried out per stem (this was due to the spread of palm trees not in one stretch) to be sampled based on existing criteria after knowing the characteristics of the population in the centers of palm sugar production that aimed to determine the areas research target areas that had a population of sugar palm plants by observing individual plant objects.

The second step was determining the sample plants that were reproducing and observing phenotypes and identifying and characterizing them using the International Plant Genetic Resources Institute (IPGRI) and the Balit Palma Guidelines. Although IPGRI has not yet issued guidelines for palm plant description, the IPGRI description of coconut plants which is a coconut one family with palm trees as a guide in the identification and characterization of palm plants [7].

The identified sample plants were the sugar palm plants. The plants were in productive condition. Identifications were included:

a. Flowers: counted the number of male mayang, the length of the male mayang series, the length of the stalk and the circumference of the male mayang; number of female mayang, length of series of female mayang, length and girth of the female stalk, the number of fruits in physiologically mature female.

b. Fruit component: calculated weight of whole fruit, length and width of fruit, number of seeds per fruit, length and width of seeds.

\section{RESULTS AND DISCUSSION}

Sugar palm in Banten Province have not been cultivated specifically. Sugar palm that is scattered in several areas of Banten, especially in the District of Serang, Pandeglang Regency is still allowed to grow wild while the utilization of palm sugar plants is so large. As an illustration of flower, fruit and seed data in Serang District, it was found in the locations of Mancak sub-district (Samples TS1-6), Gunung Sari District (TS7-TS12), and Ciomas District (TS13-TS18). Pandegelang district data was obtained in the District of Cigeulis (TS1924), District of Cinggu (TS25-TS30), District of Cibaliung (TS31 - TS36)

The results of research conducted specifically in the Pandeglang and Serang districts, showed the diversity of phenotypic characters from various palm plants.

Characterization of sugar palm flowers and fruits in Pandegelang District and Serang District can be seen in Table 1 , while the full details related to the detailed characters contained in sugar palm fruit and fruit can be seen in Table 2 (District Pandegelang), and Table 3 (District Serang).

Palm flower is a striking yellow and monocarpic. The average fruit diameter is usually $4 \mathrm{~cm}$. They are round to oval, generally contain 3 seeds, and take more than one year to mature. Axillary inflorescences crop up singly first from above and keep downward up to the palm dies. When the palm sugar tree starts to flowering, palm sap tapping can begin, but farmers generally postpone for male flowers to appear first. Palm sap is usually tapped only from male inflorescences because female inflorescences are said to produce lower quality sap, and more fibrous female stalks need more effort to fit for tapping. Generally, the near to the ground, the male inflation position, the less sap result. Production about 5 liters of sap a day can be produced by 1 flowering.

The number of female inflorescence for both locations was the same, but for the number of male inflorescence originating from Pandeglang was less than that of Serang. Sugar palm plants originating from Pandeglang had height of female infloresence. In Pandeglang regency, the sugar palm on length of female inflorescence, circumference of female inflorescence of petiol, length of female inflorescence of petiol, number of male flower, height of male inflorescence, 
length of male inflorescence $(\mathrm{cm})$, length of male inflorescence crate, length of male fruit, and circumference of male fruit, had a higher average value compared to sugar palm from Serang regency.

Female and male fruits from Pandeglang had an average length and circumference of fruit that was longer than the Serang palm sugar, but female and male fruits from Serang were heavier than Pandeglang.

The results of the characterization of flowers in Pandeglang District are presented in Table 2. The shape of the female mayang was no difference. The colors of the mayang and the colors of the female mayang from all sample locations were dark green, the color of the stem of the green mayang was yellowish green brown. Samples that had female maye were 12 samples (TS 19, TS 20, TS 22, TS 23, TS 24, TS 25, TS 27, TS 28, TS 31, TS 34, TS 36). The sample plants which had male weights were TS 25 and TS located in Cimanggu District and TS 30, TS 33, TS 34, TS 35 were in Cibaliung District (Table 2). The number of female mayang in Pandeglang Regency was varied, the highest number of female flowers was in TS 22, which was 7 mayang. The highest number of males was TS 35, which was 5 stems (Table 2). Fruit identification with observed parameters the average fruit weight in 30 seeds, length, and the largest fruit circumference was TS 19 with a weight of $33.5 \mathrm{~g}$, a length of $5.8 \mathrm{~cm}$ and a circumference of $11.9 \mathrm{~cm}$.

TABLE 1. Characterization of sugar palm flowers and fruits in Pandegelang and Serang Districts

\begin{tabular}{|c|c|c|c|c|c|}
\hline \multicolumn{2}{|r|}{ Properties } & \multicolumn{4}{|c|}{ Districts } \\
\hline & & \multicolumn{2}{|c|}{ Pandegelang } & \multicolumn{2}{|c|}{ Serang } \\
\hline & & Range & Average & Range & Average \\
\hline \multirow{5}{*}{$\begin{array}{c}\text { Female } \\
\text { Infloscence }\end{array}$} & Number of female infloresence & $1-7$ & 3.6 & $1-7$ & 3.7 \\
\hline & Height of female infloresence $(\mathrm{cm})$ & $111-200.5$ & 141.6 & $70-160$ & 120.2 \\
\hline & Length of female infloresence (cm) & $50-100$ & 62 & $30-65$ & 48.7 \\
\hline & $\begin{array}{l}\text { Circumference of female inflorescence } \\
\text { petiol }\end{array}$ & $15-50$ & 32.8 & $15-45$ & 25.9 \\
\hline & $\begin{array}{l}\text { Length of female petiol inflorescence } \\
(\mathrm{cm})\end{array}$ & $111-200.5$ & 141.6 & $70-160$ & 120.2 \\
\hline \multirow[t]{5}{*}{ Male Infloscence) } & Number of male flower & $1-5$ & 2.2 & $1-6$ & 1.6 \\
\hline & Height of male infloresence $(\mathrm{cm})$ & $116-153$ & 129.8 & $83-147$ & 116.3 \\
\hline & Length of male infloresence $(\mathrm{cm})$ & $40-80$ & 60 & $25-80$ & 45 \\
\hline & Circumference of spikelet $(\mathrm{cm})$ & $20-25$ & 21 & $15-35$ & 22.3 \\
\hline & Length of male petiol infloresence $(\mathrm{cm})$ & $116-153$ & 129.8 & $83-147$ & 116.3 \\
\hline \multirow[t]{3}{*}{ Female Fruit } & Length of female fruit & $3-5.8$ & 4.3 & $2.7-6$ & 4.7 \\
\hline & Circumference of fruit & $7-10.9$ & 9.1 & $5.8-12$ & 9.2 \\
\hline & Weight of female fruit] & $5-33.5$ & 16.3 & $5.3-37.8$ & 21.7 \\
\hline \multirow[t]{3}{*}{ Male Fruit } & Length of male fruit & $0,9-3.2$ & 2.2 & $0.8-2.6$ & 1.8 \\
\hline & Circumference of male fruit & $1.3-4.2$ & 2.7 & $0.4-4$ & 2.5 \\
\hline & Weight of male fruit & $0.8-1.9$ & 1.4 & $0.7-3.3$ & 1.75 \\
\hline
\end{tabular}


TABLE 2. Details of the character of the flowers and fruits of the sugar palm plant from Pandeglang District

\begin{tabular}{|c|c|c|c|c|c|c|c|c|c|c|c|c|c|c|c|c|c|c|}
\hline \multirow{2}{*}{ Properties } & \multicolumn{18}{|c|}{ Sample codes } \\
\hline & TS 19 & TS 20 & TS 21 & TS 22 & TS 23 & TS 24 & TS 25 & TS 26 & TS 27 & TS 28 & TS 29 & TS 30 & TS 31 & TS 32 & TS 33 & TS 34 & TS 35 & TS 36 \\
\hline $\begin{array}{l}\text { Number of female } \\
\text { flower }\end{array}$ & 1 & 1 & 4 & 7 & 5 & 4 & 1 & 3 & 6 & 3 & 0 & 0 & 5 & 3 & 4 & 4 & 0 & 3 \\
\hline $\begin{array}{l}\text { Length of female } \\
\text { infloresence }(\mathrm{cm})\end{array}$ & 80 & 50 & 70 & 60 & 57 & 40 & 70 & 82 & 65 & 25 & 0 & 0 & 50 & 75 & 100 & 56 & 0 & 80 \\
\hline $\begin{array}{l}\text { Circumference of } \\
\text { female inflorescence } \\
\text { petiol }\end{array}$ & 30 & 25 & 30 & 40 & 44 & 35 & 28 & 25 & 15 & 28 & $\mathbf{0}$ & $\mathbf{0}$ & 40 & 47 & 25 & 30 & $\mathbf{0}$ & 30 \\
\hline $\begin{array}{l}\text { Length of female } \\
\text { petiol inflorescence } \\
\text { (cm) }\end{array}$ & 143 & 123 & 113,5 & 162 & 166 & 111 & 140 & 150 & 98 & 122 & $\mathbf{0}$ & $\mathbf{0}$ & 141,5 & 164 & 147 & 120 & $\mathbf{0}$ & 200,5 \\
\hline $\begin{array}{l}\text { Number of male } \\
\text { flower }\end{array}$ & 0 & 0 & 0 & $\mathbf{0}$ & $\mathbf{0}$ & 0 & 2 & 0 & 0 & $\mathbf{0}$ & 1 & 1 & 0 & 0 & 2 & 1 & 5 & 0 \\
\hline $\begin{array}{l}\text { Length of male } \\
\text { infloresence }(\mathrm{cm})\end{array}$ & $\mathbf{0}$ & $\mathbf{0}$ & $\mathbf{0}$ & $\mathbf{0}$ & $\mathbf{0}$ & $\mathbf{0}$ & 70 & 0 & $\mathbf{0}$ & $\mathbf{0}$ & 60 & 40 & $\mathbf{0}$ & $\mathbf{0}$ & 50 & 63 & 40 & 0 \\
\hline $\begin{array}{l}\text { Circumference of } \\
\text { male inflorescence } \\
\text { petiol }(\mathrm{cm})\end{array}$ & 0 & 0 & $\mathbf{0}$ & 0 & 0 & $\mathbf{0}$ & 25 & $\mathbf{0}$ & $\mathbf{0}$ & $\mathbf{0}$ & 20 & 20 & $\mathbf{0}$ & $\mathbf{0}$ & 20 & 20 & 20 & 0 \\
\hline $\begin{array}{l}\text { Length of female } \\
\text { petiol inflorescence } \\
\text { (cm) }\end{array}$ & 0 & 0 & 0 & 0 & 0 & 0 & 116 & 0 & $\mathbf{0}$ & $\mathbf{0}$ & 138 & 140 & 0 & 0 & 120 & 101 & 153 & 0 \\
\hline $\begin{array}{l}\text { Lenght of female } \\
\text { fruit }\end{array}$ & 5,8 & 3,7 & 4 & 4,8 & 5,2 & 4,9 & 3,8 & 4,1 & 4 & 3,9 & $\mathbf{0}$ & $\mathbf{0}$ & 4,5 & 4,5 & 3,5 & 4,8 & $\mathbf{0}$ & 3,8 \\
\hline $\begin{array}{l}\text { Circumference of } \\
\text { female fruit }(\mathrm{cm})\end{array}$ & 11,9 & 7,7 & 8,6 & 10,3 & 10,9 & 8,2 & 7,7 & 8,9 & 8,7 & 7,2 & $\mathbf{0}$ & $\mathbf{0}$ & 9,8 & 9,3 & 8,3 & 10,2 & $\mathbf{0}$ & 8,4 \\
\hline $\begin{array}{l}\text { Weight of female } \\
\text { fruit (g) }\end{array}$ & 33,5 & 10 & 12,8 & 21 & 26,9 & 20,9 & 19,8 & 13,5 & 13,8 & 6,5 & $\mathbf{0}$ & $\mathbf{0}$ & 17,8 & 17,7 & 9,1 & 22,8 & $\mathbf{0}$ & 12,1 \\
\hline $\begin{array}{l}\text { Length of male fruit } \\
\text { (cm) }\end{array}$ & $\mathbf{0}$ & 0 & $\mathbf{0}$ & 0 & 0 & 0 & 3,2 & 0 & $\mathbf{0}$ & $\mathbf{0}$ & 3 & 0,9 & 0 & 0 & 2,4 & 3,2 & 1,3 & $\mathbf{0}$ \\
\hline $\begin{array}{l}\text { Circumference of } \\
\text { male fruit }(\mathrm{cm})\end{array}$ & $\mathbf{0}$ & $\mathbf{0}$ & $\mathbf{0}$ & $\mathbf{0}$ & $\mathbf{0}$ & $\mathbf{0}$ & 2,9 & $\mathbf{0}$ & $\mathbf{0}$ & $\mathbf{0}$ & 7 & 1,3 & $\mathbf{0}$ & $\mathbf{0}$ & 4,2 & 3 & 2 & $\mathbf{0}$ \\
\hline $\begin{array}{l}\text { Weight of male fruit } \\
\text { (cm) }\end{array}$ & $\mathbf{0}$ & $\mathbf{0}$ & $\mathbf{0}$ & $\mathbf{0}$ & $\mathbf{0}$ & $\mathbf{0}$ & 1,7 & $\mathbf{0}$ & $\mathbf{0}$ & $\mathbf{0}$ & 5 & 0,8 & $\mathbf{0}$ & $\mathbf{0}$ & 1,9 & 1,7 & 1 & $\mathbf{0}$ \\
\hline $\begin{array}{l}\text { Shape of female } \\
\text { inflorescence }\end{array}$ & $\begin{array}{l}\text { MB } \\
(\mathbf{0})\end{array}$ & $\begin{array}{c}\text { MB } \\
(\mathbf{0})\end{array}$ & $\begin{array}{c}\text { MB } \\
\text { (0) }\end{array}$ & $\begin{array}{c}\text { MB } \\
(0)\end{array}$ & $\begin{array}{l}\text { MB } \\
(0)\end{array}$ & $\begin{array}{c}\text { MB } \\
(\mathbf{0})\end{array}$ & $\begin{array}{l}\text { MB } \\
(0)\end{array}$ & $\begin{array}{l}\text { MB } \\
\text { (0) }\end{array}$ & $\begin{array}{l}\text { MB } \\
\text { (0) }\end{array}$ & $\begin{array}{l}\text { MB } \\
(0)\end{array}$ & $\begin{array}{l}\text { tda } \\
(9)\end{array}$ & $\begin{array}{l}\text { tda } \\
(9)\end{array}$ & $\begin{array}{l}\text { MB } \\
(0)\end{array}$ & $\begin{array}{l}\text { MB } \\
(0)\end{array}$ & $\begin{array}{l}\text { MB } \\
(0)\end{array}$ & $\begin{array}{l}\text { MB } \\
(0)\end{array}$ & $\begin{array}{l}\text { tda } \\
(9)\end{array}$ & $\begin{array}{l}\text { MB } \\
\text { (0) }\end{array}$ \\
\hline $\begin{array}{l}\text { Colour of female } \\
\text { inflorescence }\end{array}$ & $\begin{array}{l}\text { HT } \\
(1)\end{array}$ & $\begin{array}{l}\text { HT } \\
(1)\end{array}$ & $\begin{array}{c}\text { HT } \\
(1)\end{array}$ & $\begin{array}{l}\text { HT } \\
(\mathbf{1})\end{array}$ & $\begin{array}{l}\text { HT } \\
(1)\end{array}$ & $\begin{array}{c}\text { HM } \\
(0)\end{array}$ & $\begin{array}{l}\text { HT } \\
(1)\end{array}$ & $\begin{array}{l}\text { HT } \\
(1)\end{array}$ & $\begin{array}{l}\text { HT } \\
(1)\end{array}$ & $\begin{array}{c}\text { HM } \\
\text { (0) }\end{array}$ & $\begin{array}{l}\text { tda } \\
\text { (9) }\end{array}$ & $\begin{array}{l}\text { tda } \\
(9)\end{array}$ & $\begin{array}{l}\text { HT } \\
(1)\end{array}$ & $\begin{array}{l}\text { HT } \\
\text { (1) }\end{array}$ & $\begin{array}{c}\text { HM } \\
\text { (0) }\end{array}$ & $\begin{array}{l}\text { HT } \\
\text { (1) }\end{array}$ & $\begin{array}{l}\text { tda } \\
(9)\end{array}$ & $\begin{array}{l}\text { HT } \\
(1)\end{array}$ \\
\hline $\begin{array}{l}\text { Colour of female } \\
\text { inflorescence petiol }\end{array}$ & $\begin{array}{l}\text { CK } \\
(\mathbf{0})\end{array}$ & $\begin{array}{l}\text { CH } \\
(1)\end{array}$ & $\begin{array}{l}\text { CK } \\
(0)\end{array}$ & $\begin{array}{l}\text { CK } \\
(\mathbf{0})\end{array}$ & $\begin{array}{l}\text { CH } \\
\text { (1) }\end{array}$ & $\begin{array}{l}\text { CK } \\
\text { (0) }\end{array}$ & $\begin{array}{l}\text { CK } \\
\text { (0) }\end{array}$ & $\begin{array}{l}\text { CH } \\
\text { (1) }\end{array}$ & $\begin{array}{l}\text { CK } \\
(\mathbf{0})\end{array}$ & $\begin{array}{l}\text { CK } \\
(0)\end{array}$ & $\begin{array}{l}\text { tda } \\
(9)\end{array}$ & $\begin{array}{l}\text { tda } \\
(9)\end{array}$ & $\begin{array}{l}\text { CK } \\
\text { (0) }\end{array}$ & $\begin{array}{l}\text { CK } \\
\text { (0) }\end{array}$ & $\begin{array}{l}\text { CK } \\
\text { (0) }\end{array}$ & $\begin{array}{l}\text { CK } \\
\text { (0) }\end{array}$ & $\begin{array}{l}\text { tda } \\
(9)\end{array}$ & $\begin{array}{l}\text { CK } \\
(\mathbf{0})\end{array}$ \\
\hline $\begin{array}{l}\text { Colour of of female } \\
\text { infloresence }\end{array}$ & $\begin{array}{l}\text { HT } \\
(3)\end{array}$ & $\begin{array}{l}\text { HT } \\
(3)\end{array}$ & $\begin{array}{l}\text { HT } \\
\text { (3) }\end{array}$ & $\begin{array}{l}\text { HT } \\
(3)\end{array}$ & $\begin{array}{l}\text { HT } \\
(3)\end{array}$ & $\begin{array}{l}\text { HM } \\
\text { (2) }\end{array}$ & $\begin{array}{l}\text { HT } \\
(3)\end{array}$ & $\begin{array}{l}\text { HT } \\
(3)\end{array}$ & $\begin{array}{l}\text { HT } \\
(3)\end{array}$ & $\begin{array}{l}\text { HM } \\
(2)\end{array}$ & $\begin{array}{l}\text { tda } \\
\text { (9) }\end{array}$ & $\begin{array}{l}\text { tda } \\
(9)\end{array}$ & $\begin{array}{l}\text { HT } \\
\text { (3) }\end{array}$ & $\begin{array}{l}\text { HT } \\
(3)\end{array}$ & $\begin{array}{l}\text { HM } \\
(2)\end{array}$ & $\begin{array}{l}\text { HT } \\
(3)\end{array}$ & $\begin{array}{l}\text { tda } \\
\text { (9) }\end{array}$ & $\begin{array}{l}\text { HT } \\
(3)\end{array}$ \\
\hline $\begin{array}{l}\text { Shape of male } \\
\text { inflorescence }\end{array}$ & $\begin{array}{l}\text { tda } \\
(9)\end{array}$ & $\begin{array}{c}\text { tda } \\
(9)\end{array}$ & $\begin{array}{l}\text { tda } \\
\text { (9) }\end{array}$ & $\begin{array}{l}\text { tda } \\
(9)\end{array}$ & $\begin{array}{l}\text { tda } \\
(9)\end{array}$ & $\begin{array}{l}\text { tda } \\
(9)\end{array}$ & $\begin{array}{c}\text { MH } \\
(1)\end{array}$ & $\begin{array}{l}\text { tda } \\
(9)\end{array}$ & $\begin{array}{l}\text { tda } \\
\text { (9) }\end{array}$ & $\begin{array}{l}\text { tda } \\
\text { (9) }\end{array}$ & $\begin{array}{c}\text { MH } \\
\text { (1) }\end{array}$ & $\begin{array}{c}\mathrm{MC} \\
(2)\end{array}$ & $\begin{array}{l}\text { tda } \\
(9)\end{array}$ & $\begin{array}{l}\text { tda } \\
(9)\end{array}$ & $\begin{array}{c}\mathrm{MC} \\
(2)\end{array}$ & $\begin{array}{l}\text { MK } \\
(0)\end{array}$ & $\begin{array}{c}\mathrm{MC} \\
(2)\end{array}$ & $\begin{array}{l}\text { tda } \\
\text { (9) }\end{array}$ \\
\hline $\begin{array}{l}\text { Colour of male } \\
\text { inflorescence }\end{array}$ & $\begin{array}{l}\text { tda } \\
(9)\end{array}$ & $\begin{array}{l}\text { tda } \\
(9)\end{array}$ & $\begin{array}{l}\text { tda } \\
(9)\end{array}$ & $\begin{array}{l}\text { tda } \\
(9)\end{array}$ & $\begin{array}{l}\text { tda } \\
\text { (9) }\end{array}$ & $\begin{array}{l}\text { tda } \\
(9)\end{array}$ & $\begin{array}{l}\text { HT } \\
(1)\end{array}$ & $\begin{array}{l}\text { tda } \\
\text { (9) }\end{array}$ & $\begin{array}{l}\text { tda } \\
(9)\end{array}$ & $\begin{array}{l}\text { tda } \\
\text { (9) }\end{array}$ & $\begin{array}{l}\text { HT } \\
(1)\end{array}$ & $\begin{array}{c}\text { HM } \\
(\mathbf{0})\end{array}$ & $\begin{array}{l}\text { tda } \\
(9)\end{array}$ & $\begin{array}{l}\text { tda } \\
\text { (9) }\end{array}$ & $\begin{array}{c}\text { HM } \\
(\mathbf{0})\end{array}$ & $\begin{array}{l}\text { HT } \\
(1)\end{array}$ & $\underset{(\mathbf{0})}{\text { HM }}$ & $\begin{array}{l}\text { tda } \\
(9)\end{array}$ \\
\hline $\begin{array}{l}\text { Colour of male } \\
\text { inflorescence petiol }\end{array}$ & $\begin{array}{l}\text { tda } \\
(9)\end{array}$ & $\begin{array}{c}\text { tda } \\
(9)\end{array}$ & $\begin{array}{l}\text { tda } \\
\text { (9) }\end{array}$ & $\begin{array}{l}\text { tda } \\
(9)\end{array}$ & $\begin{array}{l}\text { tda } \\
(9)\end{array}$ & $\begin{array}{l}\text { tda } \\
\text { (9) }\end{array}$ & $\begin{array}{l}\text { CK } \\
\text { (0) }\end{array}$ & $\begin{array}{l}\text { tda } \\
\text { (9) }\end{array}$ & $\begin{array}{l}\text { tda } \\
\text { (9) }\end{array}$ & $\begin{array}{l}\text { tda } \\
\text { (9) }\end{array}$ & $\begin{array}{l}\text { CK } \\
\text { (0) }\end{array}$ & $\begin{array}{l}\text { CK } \\
\text { (0) }\end{array}$ & $\begin{array}{l}\text { tda } \\
(9)\end{array}$ & $\begin{array}{l}\text { tda } \\
(9)\end{array}$ & $\begin{array}{l}\text { CK } \\
(0)\end{array}$ & $\begin{array}{l}\text { CH } \\
\text { (1) }\end{array}$ & $\begin{array}{l}\text { CK } \\
(\mathbf{0})\end{array}$ & $\begin{array}{l}\text { tda } \\
\text { (9) }\end{array}$ \\
\hline $\begin{array}{l}\text { Colour of of male } \\
\text { petiol infloresence }\end{array}$ & $\begin{array}{l}\text { tda } \\
\text { (9) }\end{array}$ & $\begin{array}{c}\text { tda } \\
(9)\end{array}$ & $\begin{array}{l}\text { tda } \\
(9)\end{array}$ & $\begin{array}{l}\text { tda } \\
(9)\end{array}$ & $\begin{array}{l}\text { tda } \\
(9)\end{array}$ & $\begin{array}{l}\text { tda } \\
\text { (9) }\end{array}$ & $\begin{array}{l}\text { HT } \\
(3)\end{array}$ & $\begin{array}{l}\text { tda } \\
\text { (9) }\end{array}$ & $\begin{array}{l}\text { tda } \\
\text { (9) }\end{array}$ & $\begin{array}{l}\text { tda } \\
\text { (9) }\end{array}$ & $\begin{array}{l}\text { HM } \\
\text { (2) }\end{array}$ & $\begin{array}{c}\text { HM } \\
(2)\end{array}$ & $\begin{array}{l}\text { tda } \\
(9)\end{array}$ & $\begin{array}{l}\text { tda } \\
(9)\end{array}$ & $\begin{array}{l}\text { HM } \\
(2)\end{array}$ & $\begin{array}{l}\text { HT } \\
\text { (3) }\end{array}$ & $\begin{array}{c}\text { HM } \\
(2)\end{array}$ & $\begin{array}{l}\text { tda } \\
\text { (9) }\end{array}$ \\
\hline $\begin{array}{l}\text { Shape of female } \\
\text { fruit }\end{array}$ & BS & BS & BS & BS & BS & BS & BS & BS & BS & BS & $\begin{array}{l}\text { tda } \\
(9)\end{array}$ & $\begin{array}{l}\text { tda } \\
(9)\end{array}$ & BS & BS & BS & BS & $\begin{array}{l}\text { tda } \\
\text { (9) }\end{array}$ & BS \\
\hline $\begin{array}{l}\text { Colour of female } \\
\text { fruit }\end{array}$ & $\begin{array}{l}\text { HT } \\
(1)\end{array}$ & $\begin{array}{c}\text { HM } \\
\text { (0) }\end{array}$ & $\begin{array}{c}\text { HM } \\
\text { (0) }\end{array}$ & $\begin{array}{l}\text { HT } \\
(1)\end{array}$ & $\begin{array}{l}\text { HT } \\
(1)\end{array}$ & $\begin{array}{l}\text { HT } \\
\text { (1) }\end{array}$ & $\begin{array}{l}\text { HT } \\
(1)\end{array}$ & $\begin{array}{l}\text { HM } \\
\text { (0) }\end{array}$ & $\begin{array}{c}\text { HM } \\
(0)\end{array}$ & $\begin{array}{c}\text { HM } \\
(0)\end{array}$ & $\begin{array}{l}\text { tda } \\
(9)\end{array}$ & $\begin{array}{l}\text { tda } \\
(9)\end{array}$ & $\begin{array}{l}\text { HT } \\
(1)\end{array}$ & $\begin{array}{l}\text { HT } \\
(1)\end{array}$ & $\begin{array}{c}\text { HM } \\
(0)\end{array}$ & $\begin{array}{l}\text { HT } \\
(1)\end{array}$ & $\begin{array}{l}\text { tda } \\
\text { (9) }\end{array}$ & $\begin{array}{l}\text { HM } \\
\text { (0) }\end{array}$ \\
\hline Shape of male fruit & $\begin{array}{l}\text { tda } \\
(9)\end{array}$ & $\begin{array}{c}\text { tda } \\
(9)\end{array}$ & $\begin{array}{l}\text { tda } \\
(9)\end{array}$ & $\begin{array}{l}\text { tda } \\
(9)\end{array}$ & $\begin{array}{l}\text { tda } \\
(9)\end{array}$ & $\begin{array}{l}\text { tda } \\
\text { (9) }\end{array}$ & BS & $\begin{array}{l}\text { tda } \\
\text { (9) }\end{array}$ & $\begin{array}{l}\text { tda } \\
\text { (9) }\end{array}$ & $\begin{array}{l}\text { tda } \\
\text { (9) }\end{array}$ & BS & BS & $\begin{array}{l}\text { tda } \\
(9)\end{array}$ & BS & BS & & & $\begin{array}{l}\text { tda } \\
(9)\end{array}$ \\
\hline Colour of male fruit & $\begin{array}{l}\text { tda } \\
\text { (9) }\end{array}$ & $\begin{array}{l}\text { tda } \\
(9)\end{array}$ & $\begin{array}{l}\text { tda } \\
(9)\end{array}$ & $\begin{array}{l}\text { tda } \\
(9)\end{array}$ & $\begin{array}{l}\text { tda } \\
(9)\end{array}$ & $\begin{array}{l}\text { tda } \\
\text { (9) }\end{array}$ & $\begin{array}{c}\text { HM } \\
\text { (0) }\end{array}$ & $\begin{array}{l}\text { tda } \\
\text { (9) }\end{array}$ & $\begin{array}{l}\text { tda } \\
\text { (9) }\end{array}$ & $\begin{array}{l}\text { tda } \\
\text { (9) }\end{array}$ & $\begin{array}{c}\text { HM } \\
\text { (0) }\end{array}$ & $\begin{array}{c}\text { HM } \\
(0)\end{array}$ & $\begin{array}{l}\text { tda } \\
(9)\end{array}$ & $\begin{array}{l}\text { tda } \\
\text { (9) }\end{array}$ & $\begin{array}{c}\text { HM } \\
(\mathbf{0})\end{array}$ & $\begin{array}{c}\text { HM } \\
(\mathbf{0})\end{array}$ & $\underset{\text { (0) }}{\text { HM }}$ & $\begin{array}{l}\text { tda } \\
\text { (9) }\end{array}$ \\
\hline
\end{tabular}


TABLE 3. Details of the character of the flowers and fruits of the sugar palm plant from Serang District

\begin{tabular}{|c|c|c|c|c|c|c|c|c|c|c|c|c|c|c|c|c|c|c|}
\hline \multirow{2}{*}{ Properties } & \multicolumn{18}{|c|}{ Sample codes } \\
\hline & IS 1 & Is 2 & TS 3 & TS 4 & TS 5 & TS 6 & IS 7 & TS 8 & IS 9 & TS 10 & TS 11 & TS 12 & TS 13 & TS 14 & TS 15 & TS 16 & TS 17 & S 18 \\
\hline $\begin{array}{l}\text { Number of female } \\
\text { flower }\end{array}$ & 1 & 1 & 7 & 6 & 5 & 5 & 6 & 1 & 3 & 7 & 3 & 1 & 2 & 5 & 5 & 1 & 7 & 1 \\
\hline $\begin{array}{l}\text { Length of female } \\
\text { infloresence (cm) }\end{array}$ & 40 & 60 & 30 & 55 & 55 & 40 & 45 & 30 & 60 & 45 & 65 & 50 & 40 & 55 & 55 & 56 & 40 & 46 \\
\hline $\begin{array}{l}\text { Circumference of } \\
\text { female inflorescence } \\
\text { petiol }\end{array}$ & 28 & 40 & 18 & 23 & 30 & 26 & 20 & 15 & 25 & 20 & 45 & 25 & 30 & 20 & 18 & 20 & 40 & 23 \\
\hline $\begin{array}{l}\text { Length of female } \\
\text { petiol inflorescence } \\
\text { (cm) }\end{array}$ & 70 & 130 & 160 & 146 & 139 & 113 & 150 & 150 & 150 & 95 & 105 & 137 & 71 & 120 & 114 & 103 & 107 & 103 \\
\hline $\begin{array}{l}\text { Number of male } \\
\text { flower }\end{array}$ & 6 & 1 & $\mathbf{0}$ & 0 & 1 & 1 & 0 & 1 & 1 & 0 & 0 & 0 & o & 0 & $\mathbf{0}$ & 1 & 1 & 1 \\
\hline $\begin{array}{l}\text { Length of male } \\
\text { infloresence }(\mathbf{c m})\end{array}$ & 50 & 40 & 0 & $\mathbf{0}$ & 45 & 50 & o & 25 & 30 & o & o & 0 & o & 0 & o & 47 & 80 & 38 \\
\hline $\begin{array}{l}\text { Circumference of } \\
\text { male inflorescence } \\
\text { petiol }(\mathbf{c m})\end{array}$ & 20 & 35 & 0 & 0 & 30 & 22 & 0 & 15 & 15 & 0 & 0 & 0 & $\mathbf{0}$ & 0 & $\mathbf{0}$ & 23 & 20 & 21 \\
\hline $\begin{array}{l}\text { Length of female } \\
\text { petiol inflorescence } \\
\text { (cm) }\end{array}$ & 127 & 110 & 0 & 0 & 124 & 120 & 0 & 135 & 147 & 0 & 0 & 0 & 0 & 0 & $\mathbf{0}$ & 106 & 95 & 83 \\
\hline $\begin{array}{l}\text { Lenght of female } \\
\text { fruit }\end{array}$ & 4,8 & 5,2 & 5,6 & 4,3 & 5,1 & 5,9 & 5,7 & 6 & 2,7 & 3,1 & 2,8 & 5,5 & 5,9 & 5,2 & 4,4 & 3,8 & 3,2 & 5,7 \\
\hline $\begin{array}{l}\text { Circumference of } \\
\text { female fruit }(\mathrm{cm})\end{array}$ & 9,1 & 8,6 & 11,7 & 8,8 & 10,5 & 12 & 11,2 & 11,6 & 5,8 & 7,2 & 1,6 & 11 & 10,5 & 9,6 & 9 & 8,7 & 7,4 & 12 \\
\hline $\begin{array}{l}\text { Weight of female } \\
\text { fruit (g) }\end{array}$ & 26,2 & 21,5 & 28,8 & 14,5 & 25,7 & 37,8 & 29,7 & 32,2 & 5,3 & 7,6 & 3,3 & 29,8 & 31,3 & 22,9 & 17,1 & 12,7 & 6,9 & 37,5 \\
\hline $\begin{array}{l}\text { Length of male fruit } \\
\text { (cm) }\end{array}$ & 0,8 & 1,4 & 0 & 0 & 1,5 & 2,2 & $\mathbf{0}$ & 2,2 & 1,6 & 0 & 0 & 0 & 0 & 0 & 0 & 0,6 & 2,6 & 1,5 \\
\hline $\begin{array}{l}\text { Circumference of } \\
\text { male fruit }(\mathbf{c m})\end{array}$ & 2,7 & 3,2 & 0 & 0 & 3,3 & 4 & 0 & 0,6 & 0,4 & 0 & 0 & 0 & o & 0 & $\mathbf{0}$ & 3,6 & 3,4 & 1,5 \\
\hline $\begin{array}{l}\text { Weight of male fruit } \\
\text { (cm) }\end{array}$ & 0,7 & 1,3 & 0 & 0 & 1,5 & 2,4 & 0 & 1,6 & 1,2 & 0 & $\mathbf{0}$ & $\mathbf{0}$ & $\mathbf{0}$ & 0 & $\mathbf{0}$ & 3,3 & 2,9 & 0,83 \\
\hline $\begin{array}{l}\text { Shape of female } \\
\text { inflorescence }\end{array}$ & $\begin{array}{c}\text { MB } \\
(\mathbf{0})\end{array}$ & $\begin{array}{c}\text { MB } \\
(\mathbf{0})\end{array}$ & $\begin{array}{c}\text { MB } \\
\text { (0) }\end{array}$ & $\begin{array}{c}\text { MB } \\
(\mathbf{0})\end{array}$ & $\begin{array}{c}\text { MB } \\
(\mathbf{0})\end{array}$ & $\begin{array}{c}\text { MB } \\
(\mathbf{0})\end{array}$ & $\begin{array}{c}\text { MB } \\
\text { (0) }\end{array}$ & $\begin{array}{c}\text { MB } \\
(0)\end{array}$ & $\begin{array}{l}\text { MB } \\
(\mathbf{0})\end{array}$ & $\begin{array}{c}\text { MB } \\
\text { (0) }\end{array}$ & $\begin{array}{c}\text { MB } \\
(0)\end{array}$ & $\begin{array}{c}\text { MB } \\
(0)\end{array}$ & $\begin{array}{c}\text { MB } \\
(\mathbf{0})\end{array}$ & $\begin{array}{c}\text { MB } \\
\text { (0) }\end{array}$ & $\begin{array}{c}\text { MB } \\
(\mathbf{0})\end{array}$ & $\begin{array}{c}\text { MB } \\
(0)\end{array}$ & $\begin{array}{c}\text { MB } \\
(\mathbf{0})\end{array}$ & $\begin{array}{l}\text { MB } \\
(\mathbf{0})\end{array}$ \\
\hline $\begin{array}{l}\text { Colour of female } \\
\text { inflorescence }\end{array}$ & $\begin{array}{c}\text { HM } \\
(\mathbf{0})\end{array}$ & $\underset{(0)}{\text { HM }}$ & $\begin{array}{l}\text { HT } \\
\text { (1) }\end{array}$ & $\begin{array}{l}\text { HT } \\
\text { (1) }\end{array}$ & $\begin{array}{l}\text { HT } \\
\text { (1) }\end{array}$ & $\begin{array}{l}\mathrm{HT} \\
(\mathbf{1})\end{array}$ & $\begin{array}{c}\text { HM } \\
\text { (0) }\end{array}$ & $\begin{array}{l}\text { HM } \\
(0)\end{array}$ & $\begin{array}{l}\text { HT } \\
\text { (1) }\end{array}$ & $\begin{array}{c}\text { HM } \\
\text { (0) }\end{array}$ & $\begin{array}{l}\text { HT } \\
\text { (1) }\end{array}$ & $\begin{array}{l}\text { HT } \\
\text { (1) }\end{array}$ & $\begin{array}{l}\text { HT } \\
\text { (1) }\end{array}$ & $\begin{array}{l}\text { HT } \\
\text { (1) }\end{array}$ & $\begin{array}{l}\text { HM } \\
\text { (0) }\end{array}$ & $\begin{array}{l}\text { HT } \\
\text { (1) }\end{array}$ & $\begin{array}{l}\text { HM } \\
\text { (0) }\end{array}$ & $\begin{array}{l}\text { HT } \\
\text { (1) }\end{array}$ \\
\hline $\begin{array}{l}\text { Colour of female } \\
\text { inflorescence petiol }\end{array}$ & $\begin{array}{l}\text { CK } \\
(\mathbf{0})\end{array}$ & $\begin{array}{l}\text { CK } \\
(\mathbf{0})\end{array}$ & $\begin{array}{l}\text { CK } \\
\text { (0) }\end{array}$ & $\begin{array}{l}\text { CH } \\
\text { (1) }\end{array}$ & $\begin{array}{l}\text { CH } \\
\text { (1) }\end{array}$ & $\underset{(1)}{\mathrm{CH}}$ & $\begin{array}{l}\mathrm{CH} \\
(\mathbf{1})\end{array}$ & $\begin{array}{l}\mathrm{CH} \\
\text { (1) }\end{array}$ & $\begin{array}{l}\mathrm{CH} \\
\text { (1) }\end{array}$ & $\begin{array}{l}\text { CH } \\
\text { (1) }\end{array}$ & $\begin{array}{l}\mathrm{CH} \\
(\mathbf{1})\end{array}$ & $\begin{array}{l}\mathrm{CH} \\
\text { (1) }\end{array}$ & $\begin{array}{l}\mathrm{CH} \\
\text { (1) }\end{array}$ & $\begin{array}{l}\text { CH } \\
\text { (1) }\end{array}$ & $\begin{array}{l}\text { CK } \\
\text { (0) }\end{array}$ & $\begin{array}{l}\mathrm{CH} \\
\text { (1) }\end{array}$ & $\begin{array}{l}\mathrm{CH} \\
\text { (1) }\end{array}$ & $\begin{array}{l}\text { CK } \\
\text { (0) }\end{array}$ \\
\hline $\begin{array}{l}\text { Colour of of female } \\
\text { infloresence }\end{array}$ & $\begin{array}{c}\text { HM } \\
(2)\end{array}$ & $\begin{array}{r}\mathrm{HM} \\
(2)\end{array}$ & $\begin{array}{l}\text { HG } \\
(1)\end{array}$ & $\begin{array}{l}\text { HT } \\
\text { (3) }\end{array}$ & $\begin{array}{l}\text { HT } \\
(3)\end{array}$ & $\begin{array}{l}\text { HT } \\
\text { (3) }\end{array}$ & $\begin{array}{c}\mathrm{HM} \\
(2)\end{array}$ & $\begin{array}{l}\text { HM } \\
(2)\end{array}$ & $\begin{array}{l}\text { HG } \\
\text { (1) }\end{array}$ & $\begin{array}{c}\mathrm{HM} \\
(2)\end{array}$ & $\begin{array}{l}\text { HT } \\
\text { (3) }\end{array}$ & $\begin{array}{l}\text { HT } \\
\text { (3) }\end{array}$ & $\begin{array}{l}\text { HG } \\
\text { (1) }\end{array}$ & $\begin{array}{l}\text { HG } \\
(1)\end{array}$ & $\begin{array}{c}\mathrm{HM} \\
(2)\end{array}$ & $\begin{array}{c}\mathrm{HM} \\
(2)\end{array}$ & $\begin{array}{c}\mathrm{HM} \\
(2)\end{array}$ & $\begin{array}{l}\text { HT } \\
(3)\end{array}$ \\
\hline $\begin{array}{l}\text { Shape of male } \\
\text { inflorescence }\end{array}$ & $\begin{array}{c}\text { MB } \\
(\mathbf{0})\end{array}$ & $\begin{array}{c}\text { MB } \\
(\mathbf{0})\end{array}$ & $\begin{array}{l}\text { tda } \\
(9)\end{array}$ & $\begin{array}{l}\text { tda } \\
\text { (9) }\end{array}$ & $\begin{array}{c}\text { MB } \\
(\mathbf{0})\end{array}$ & $\begin{array}{l}\text { MK } \\
\text { (0) }\end{array}$ & $\begin{array}{l}\text { tda } \\
(9)\end{array}$ & $\underset{(1)}{\text { MH }}$ & $\begin{array}{c}\text { MH } \\
(1)\end{array}$ & $\begin{array}{l}\text { tda } \\
(9)\end{array}$ & $\begin{array}{l}\text { tda } \\
(9)\end{array}$ & $\begin{array}{l}\text { tda } \\
\text { (9) }\end{array}$ & $\begin{array}{l}\text { tda } \\
(9)\end{array}$ & $\begin{array}{l}\text { tda } \\
(9)\end{array}$ & $\begin{array}{l}\text { tda } \\
(9)\end{array}$ & $\begin{array}{l}\text { MK } \\
(0)\end{array}$ & $\begin{array}{l}\text { MK } \\
\text { (0) }\end{array}$ & $\begin{array}{l}\text { MK } \\
(\mathbf{0})\end{array}$ \\
\hline $\begin{array}{l}\text { Colour of male } \\
\text { inflorescence }\end{array}$ & $\begin{array}{c}\text { HM } \\
(\mathbf{0})\end{array}$ & $\begin{array}{r}\text { HM } \\
(0)\end{array}$ & $\begin{array}{l}\text { tda } \\
(9)\end{array}$ & $\begin{array}{l}\text { tda } \\
(9)\end{array}$ & $\begin{array}{r}\text { HM } \\
(\mathbf{0})\end{array}$ & $\begin{array}{c}\mathrm{HM} \\
(\mathbf{0})\end{array}$ & $\begin{array}{l}\text { tda } \\
(9)\end{array}$ & $\begin{array}{c}\text { HM } \\
(0)\end{array}$ & $\begin{array}{l}\text { HT } \\
\text { (1) }\end{array}$ & $\begin{array}{l}\text { tda } \\
(9)\end{array}$ & $\begin{array}{l}\text { tda } \\
(9)\end{array}$ & $\begin{array}{l}\text { tda } \\
\text { (9) }\end{array}$ & $\begin{array}{l}\text { tda } \\
(9)\end{array}$ & $\begin{array}{l}\text { tda } \\
(9)\end{array}$ & $\begin{array}{l}\text { tda } \\
(9)\end{array}$ & $\begin{array}{l}\text { HM } \\
\text { (0) }\end{array}$ & $\begin{array}{c}\text { HM } \\
\text { (0) }\end{array}$ & $\begin{array}{r}\text { HM } \\
(0)\end{array}$ \\
\hline $\begin{array}{l}\text { Colour of male } \\
\text { inflorescence petiol }\end{array}$ & $\begin{array}{l}\mathrm{CH} \\
(1)\end{array}$ & $\begin{array}{l}\mathrm{CH} \\
\text { (1) }\end{array}$ & $\begin{array}{l}\text { tda } \\
(9)\end{array}$ & $\begin{array}{l}\text { tda } \\
(9)\end{array}$ & $\begin{array}{l}\mathrm{CH} \\
\text { (1) }\end{array}$ & $\underset{(1)}{\mathrm{CH}}$ & $\begin{array}{l}\text { tda } \\
(9)\end{array}$ & $\begin{array}{l}\text { CK } \\
(\mathbf{0})\end{array}$ & $\begin{array}{l}\text { CK } \\
(\mathbf{0})\end{array}$ & $\begin{array}{l}\text { tda } \\
(9)\end{array}$ & $\begin{array}{l}\text { tda } \\
(9)\end{array}$ & $\begin{array}{l}\text { tda } \\
\text { (9) }\end{array}$ & $\begin{array}{l}\text { tda } \\
(9)\end{array}$ & $\begin{array}{l}\text { tda } \\
(9)\end{array}$ & $\begin{array}{l}\text { tda } \\
(9)\end{array}$ & $\begin{array}{l}\mathrm{CH} \\
\text { (1) }\end{array}$ & $\begin{array}{l}\text { CK } \\
\text { (0) }\end{array}$ & $\begin{array}{l}\text { CK } \\
\text { (0) }\end{array}$ \\
\hline $\begin{array}{l}\text { Colour of of male } \\
\text { petiol infloresence }\end{array}$ & $\begin{array}{c}\text { HM } \\
(2)\end{array}$ & $\begin{array}{l}\text { HM } \\
(2)\end{array}$ & $\begin{array}{l}\text { tda } \\
(9)\end{array}$ & $\begin{array}{l}\text { tda } \\
\text { (9) }\end{array}$ & $\begin{array}{l}\text { HM } \\
(2)\end{array}$ & $\begin{array}{c}\text { HM } \\
(2)\end{array}$ & $\begin{array}{l}\text { tda } \\
(9)\end{array}$ & $\begin{array}{l}\text { HM } \\
(2)\end{array}$ & $\begin{array}{l}\text { HM } \\
(2)\end{array}$ & $\begin{array}{l}\text { tda } \\
(9)\end{array}$ & $\begin{array}{l}\text { tda } \\
(9)\end{array}$ & $\begin{array}{l}\text { tda } \\
\text { (9) }\end{array}$ & $\begin{array}{l}\text { tda } \\
\text { (9) }\end{array}$ & $\begin{array}{l}\text { tda } \\
\text { (9) }\end{array}$ & $\begin{array}{l}\text { tda } \\
(9)\end{array}$ & $\begin{array}{l}\text { HT } \\
(3)\end{array}$ & $\begin{array}{l}\text { HM } \\
(2)\end{array}$ & $\begin{array}{l}\text { HM } \\
(2)\end{array}$ \\
\hline Shape of female fruit & BS & BS & BS & BS & BS & BS & BS & BS & BS & BS & BS & BS & BS & BS & BS & BS & BS & BS \\
\hline $\begin{array}{l}\text { Colour of female } \\
\text { fruit }\end{array}$ & $\begin{array}{l}\text { HT } \\
\text { (1) }\end{array}$ & $\begin{array}{r}\text { HM } \\
(\mathbf{0})\end{array}$ & $\begin{array}{c}\text { HM } \\
(\mathbf{0})\end{array}$ & $\begin{array}{l}\text { HT } \\
\text { (1) }\end{array}$ & $\begin{array}{l}\text { HT } \\
\text { (1) }\end{array}$ & $\begin{array}{l}\text { HT } \\
\text { (1) }\end{array}$ & $\begin{array}{l}\text { HT } \\
(\mathbf{1})\end{array}$ & $\begin{array}{l}\text { HM } \\
(\mathbf{0})\end{array}$ & $\begin{array}{l}\text { HM } \\
(\mathbf{0})\end{array}$ & $\begin{array}{c}\text { HM } \\
\text { (0) }\end{array}$ & $\begin{array}{l}\text { HT } \\
\text { (1) }\end{array}$ & $\begin{array}{c}\mathrm{HM} \\
\text { (0) }\end{array}$ & $\begin{array}{l}\text { HT } \\
\text { (1) }\end{array}$ & $\begin{array}{r}\text { HT } \\
\text { (1) }\end{array}$ & $\begin{array}{c}\text { HM } \\
\text { (0) }\end{array}$ & $\begin{array}{l}\text { HT } \\
\text { (1) }\end{array}$ & $\begin{array}{c}\mathrm{HM} \\
(\mathbf{0})\end{array}$ & $\begin{array}{l}\text { HM } \\
(\mathbf{0})\end{array}$ \\
\hline Shape of male fruit & BS & BS & $\begin{array}{l}\text { tda } \\
(9)\end{array}$ & $\begin{array}{l}\text { tda } \\
(9)\end{array}$ & BS & BS & $\begin{array}{l}\text { tda } \\
(9)\end{array}$ & BS & BS & $\begin{array}{l}\text { tda } \\
(9)\end{array}$ & $\begin{array}{l}\text { tda } \\
(9)\end{array}$ & $\begin{array}{l}\text { tda } \\
\text { (9) }\end{array}$ & $\begin{array}{l}\text { tda } \\
(9)\end{array}$ & $\begin{array}{l}\text { tda } \\
(9)\end{array}$ & $\begin{array}{l}\text { tda } \\
(9)\end{array}$ & BS & BS & BS \\
\hline Colour of male fruit & $\begin{array}{c}\text { HM } \\
\text { (0) }\end{array}$ & $\begin{array}{r}\text { HM } \\
\text { (0) }\end{array}$ & $\begin{array}{l}\text { tda } \\
(9)\end{array}$ & $\begin{array}{l}\text { tda } \\
(9)\end{array}$ & $\underset{(\mathbf{0})}{\mathrm{HM}}$ & $\begin{array}{c}\text { HM } \\
\text { (0) }\end{array}$ & $\begin{array}{l}\text { tda } \\
\text { (9) }\end{array}$ & $\begin{array}{c}\text { HM } \\
\text { (0) }\end{array}$ & $\underset{(\mathbf{0})}{\mathrm{HM}}$ & $\begin{array}{l}\text { tda } \\
\text { (9) }\end{array}$ & $\begin{array}{l}\text { tda } \\
\text { (9) }\end{array}$ & $\begin{array}{l}\text { tda } \\
\text { (9) }\end{array}$ & $\begin{array}{l}\text { tda } \\
\text { (9) }\end{array}$ & $\begin{array}{l}\text { tda } \\
\text { (9) }\end{array}$ & $\begin{array}{l}\text { tda } \\
\text { (9) }\end{array}$ & $\begin{array}{c}\text { HM } \\
\text { (0) }\end{array}$ & $\begin{array}{c}\text { HM } \\
\text { (0) }\end{array}$ & $\begin{array}{l}\text { HM } \\
\text { (0) }\end{array}$ \\
\hline
\end{tabular}




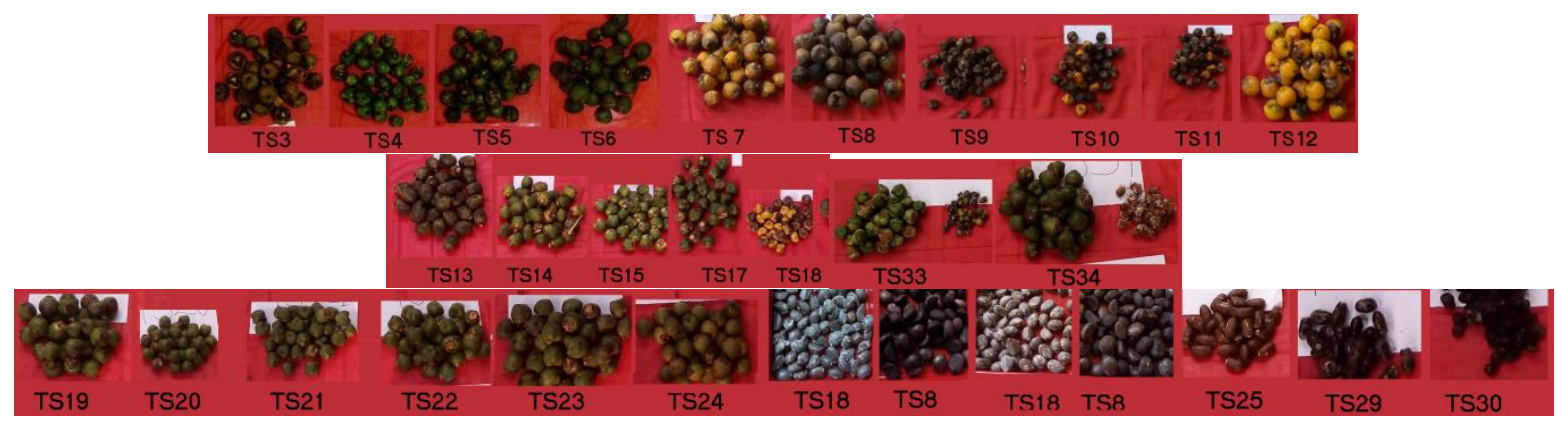

Fig. 1. The appearance of sugar palm fruit and seeds from several locations

The results of the characterization of sugar palm flowers from Serang Regency are presented in Table 3. The appearance of sugar palm fruit and seeds from the two districts can be seen in Figure 1. Every inflorescence spikelet has a little female flower at the end of the tip and coordinates male flowers throughout from there to the tip. The shape of male flowers are smaller than female flowers.

The initial inflorescence appeared from a node near the top meristem in common with 3-6 (12) other inflorescences that only growed the female flowers. Following this stage male inflorescences arised in descending order from the topmost leaf axil bellows of the female inflorescences. The upmost female inflorescences forwarded to expand for about 2 years up to the fruits are mature and fall over a period of a year after the tree lacks energy and slowly dies. Each node suffered only 1 inflorescence. Depending on the use of the sugar palm tree, tapping, and ripening, the tree can live for several years but produce smaller fruits and seeds. The performance of male inflorescences, in this case, continue the base of the tree before the tree dies. When ripe, the fruit is yellow, about $5 \mathrm{~cm}$ in diameter, with 2-3 seeds each.

According to [7] early type of sugar palm has a shorter and smaller tree than In type palm, and but the fruit and sugar palm seeds are also smaller than In type palm. Furthermore, seeds that qualify as seeds are oval shaped with a size of 25-40 x 15$25 \mathrm{~mm}$ brownish black, shiny, slippery surface, transverse incision in a somewhat triangular or round shape. Flower, fruit and seed data are not available in all locations in this study, only in certain locations. In addition, the age of the observed sugar palm tree is also unknown.

\section{CONCLUSION}

Phenotype characterization results on the flowers and fruit of the sugar palm trees in Pandeglang District with more data on the population of sugar palm trees when compared to the Serang District, The accessions used in this study showed variations in the appearance of male and female flowers and fruit from the sugar palm tree accessions.

\section{REFERENCES}

[1] Syukur, C. 2006. Pengelolaan Plasma Nutfah. Pusat Penelitaian Pengembangan Perkebunan. Lokakarya Nasional Genetik di Indonesia: Manfaat Ekonomi untuk Mewujudkan Ketahanan Nasional. Bogor.

[2] Dinas Pertanian Provinsi Banten. 2016. Angka Tetap Statistik Perkebunan.

[3] Tenda, E.T., Maskromo, I dan Heliyanto. 2010. Eksplorasi Plasma Nutfah Aren (Arengan pinnatai Merr.) di Kutai Timur Provins
Kalimantan Timur. Balai Penelitian Tanaman Kelapa dan Palma Lain. Manado. Buletin Palma No. 38

[4] Jusuf, M. 2005. Metode Ekplorasi, Inventarisasi, Evaluasi dan Konservasi Plasma Nutfah. Pusat Penelitian Bioteknologi. Institut Pertanian Bogor.

[5] Ferita, I., Tawarati., Syarif, Z. 2015. Identifikasi dan Karakterisasi Tanaman Enau (Arengan pinnata) di Kabupaten Gayo Lues. Prosiding Seminar Nasional Masyarakat Biodiversitas Indonesia. Vol. 1 Nomor 1. Hal: 31-37.

[6] Harahap, P. 2017. Eksplorasi dan Identifikasi Tanaman Aren (Arengan pinnata Merr.) di Kabupaten Tapanuli Selatan. Tesis. Universitas Sumatera Utara.

[7] IPGRI. 1995. Descriptor for Coconut. Rome Italy. 